INGENIERÍA INDUSTRIAL

\title{
Identificación de brechas tecnológicas en automatización industrial de las empresas del sector metalmecánico de Caldas, Colombia
}

\section{Technological gaps in industrial automation in metal mechanical enterprises in Caldas, Colombia}

\author{
Alex M. Ovalle*, Olga L. Ocampo*§, Maria T. Acevedo**. \\ *Universidad Autónoma de Manizales, Departamento de Mecánica y Producción. \\ **Servicio Nacional de Aprendizaje-SENA-Regional Caldas, Centro de Automatización Industrial. \\ movalle@autonoma.edu.co, §olocampo@autonoma.edu.co,mtacevedo@sena.edu.co
}

(Recibido: Octubre 17 de 2012 - Aceptado: Abril 06 de 2013)

\begin{abstract}
Resumen
La identificación de brechas tecnológicas en automatización industrial de las empresas del sector metalmecánico de Caldas fue el producto de una investigación cuantitativa, de tipo descriptivo, que abarcó la evaluación de los niveles de automatización y un análisis de las palancas de fabricación. Los instrumentos metodológicos, validados por grupo de expertos del SENA-Servicio Nacional de Aprendizaje- y de la Universidad Autónoma de Manizales, fueron aplicados a las empresas seleccionadas, pertenecientes a la mesa sectorial metalmecánica. Los niveles de automatización en el 40\% de las empresas fueron medios; el 10\% se encuentra entre la categoría media-alta a clase mundial y el 50\% restante tiene bajos niveles de automatización y una mayor brecha tecnológica, debido al carácter manual de las operaciones de la cadena de valor. Las mayores brechas se detectaron en la gestión logística que comprende las operaciones de abastecimiento de materiales, almacenamiento y despacho. Se encontraron falencias en la gestión tecnológica, en aspectos claves como la identificación y el seguimiento de nuevas tecnologías y los planes tecnológicos. Mejorar la gestión tecnológica en las organizaciones, puede contribuir al cierre de las brechas tecnológicas detectadas.
\end{abstract}

Palabras clave: Automatización industrial, brechas tecnológicas, gestión tecnológica, palancas de fabricación

\begin{abstract}
Identifying industrial automation technological gaps in metal mechanical companies of Caldas was the quantitative and descriptive research product, which included the automation level assessment and a manufacturing gearing analysis. Methodological tools, validated by expert group of the SENA (National Apprenticeship Service) and the Universidad Autonoma de Manizales, were applied to the selected companies that belong to metal mechanical sector. The $40 \%$ of companies has a medium automation level, the $10 \%$ of them is among the medium-high to world class automation level; the remaining 50\% has a higher technology gaps due to the low automation levels which were found, given the chain value manual operations. The largest gaps were detected in logistic management operations which comprise material sourcing, storage and delivery. Shortcomings in technology management in key aspects such as the identification and tracking of new technologies and technology plans were detected. Improving Technology management in organizations could help close the identified technology gaps.
\end{abstract}

Keywords: Industrial automation, technological gaps, technology management, manufacturing gearing 


\section{Introducción}

El grado de desarrollo de la cadena metalmecánica es un determinante del progreso industrial de un país, dada su estrecha vinculación con las actividades económicas de los sectores minero, energético, industrial y de construcción. Los diferentes subsectores que componen la Cadena Productiva Metalmecánica son complementarios e indispensables para el desarrollo de los sectores de construcción, eléctrico, plástico y calzado, entre otros (López, 2005). Según el DNP (Departamento Nacional de Planeación, 2007), el sector metalmecánico tiene un alto efecto multiplicador, porque los procesos conllevan una mayor tecnología y complejidad, lo cual contribuye a generar inversión, empleo altamente calificado y elaborar productos de alto valor agregado.

El liderazgo de la cadena metalmecánica a nivel mundial es producto de la asimilación de tecnologías, criterios de diseño, calidad y diferenciación del producto. En Colombia, por su parte, la cadena metalmecánica presenta características tecnológicas propias de acuerdo con el tamaño de sus empresas (SENA, 2002). Diversos estudios del sector metalmecánico realizados en Bogotá y Cundinamarca (Cámara de Comercio de Bogotá, 2005), Bucaramanga (Observatorio de Mercado de Trabajo, 2010), Pereira y Dosquebradas González et al., (2004) y Manizales (López, 2005) permiten identificar fortalezas y debilidades de la cadena. Entre las fortalezas se destacan el conocimiento y facilidad de asimilación de las tecnologías utilizadas; como debilidades se reportan la falta de innovación, la ausencia de programas de modernización tecnológica y la limitada incorporación de la automatización para garantizar una mayor productividad y calidad; aunque se han sofisticado algunos procesos, por implementación de dispositivos de control, sigue empleándose tecnología tradicional (SENA, 2002).

En consecuencia, la poca sofisticación y agregación de valor en los procesos productivos, los bajos niveles de innovación y de absorción de tecnologías, el rezago en penetración de tecnologías de información limitan la competitividad (DNP, 2009). Es necesario facilitar el desarrollo tecnológico mediante proyectos de innovación y automatización, para mejorar la competitividad, en especial de las Mipymes (DNP y Colciencias, 2005).

La estrecha relación competitividad y productividad ha llevado a que la automatización sea considerada como una estrategia y ventaja competitiva (Ruedas, 2010). La automatización industrial comprende los elementos tecnológicos, con aplicación de sistemas mecánicos, electrónicos y computarizados, que permiten operar y controlar la producción, con mínima o ninguna intervención del serhumano Moreno,(2001); Piedrahita,(2001); incluye el manejo de la información para la toma de decisiones en tiempo real, la informática y el control automatizado para la ejecución autónoma y óptima de procesos, basados en los planes de la dirección empresarial (DNP y Colciencias, 2000). En la práctica, alcanza diferentes niveles porque su implementación en los procesos industriales varía considerablemente, según la estrategia de manufactura. Dicha estrategia ha sido categorizada en los niveles infantil, medio, adulto y clase mundial, Miltenburg, (2005).

Además de la productividad, la competitividad está relacionada con múltiples capacidades o competencias esenciales, que permiten a la organización desarrollar con éxito una actividad a partir de la combinación y coordinación de los recursos disponibles, para generar ventajas competitivas Huerta et al., (2004). La Gestión de la Tecnología es una de las competencias esenciales que la organización debe desarrollar con el propósito de adquirir, adaptar, asimilar y transferir tecnologías útiles en los procesos de fabricación de productos y prestación de servicios, Ortiz \& Nagles, (2008). La Gestión tecnológica es indispensable para el desarrollo de industrias de clase mundial.

Por otra parte es necesario fomentar la innovación, la sofisticación y la agregación de valor en los procesosproductivospara eldesarrollodeindustrias 
de clase mundial. Diferentes estrategias como la investigación aplicada a la solución de problemas y el desarrollo tecnológico, entre otras, han sido propuestas por el CONPES 3582; el cual plantea que la identificación de brechas tecnológicas y el desarrollo de proyectos de investigación aplicada que mejoren la competitividad son opciones para llevar a cabo estas estrategias (DNP, 2008).

La brecha es la distancia existente entre dos o más puntos de referencia en torno a indicadores científicos, tecnológicos, comerciales, económicos, productivos, de infraestructura, de mercado, de formación de talento humano, entre otros: Medina et al., (2010). La brecha tecnológica considera por tanto, indicadores tecnológicos Medina et al (2010) y es la distancia entre la industria local y las empresas de clase mundial, Pineda \& Jara (2009). Diferentes criterios han sido empleados para su identificación Pineda \& Jara, (2009); Medina et al., (2010), los cuales incluyen aspectos de desarrollo empresarial, tecnológico e investigación, inversión, capital físico y humano y políticas de competitividad. Desde el punto de vista de un sistema de producción, la brecha puede ser analizada considerando el nivel de capacidad global, equivalente a la suma de las capacidades de las palancas de fabricación: talento humano, estructura y control, gestión logística, gestión de la producción, gestión tecnológica e infraestructura Miltenburg, (2005).

El nivel de capacidad global de la organización fue considerado para la identificación de brechas tecnológicas en automatización industrial de las empresas del sector metalmecánico del departamento de Caldas, Colombia; donde se abordaron aspectos como la evaluación de los niveles de automatización existentes, el análisis de los aspectos de gestión tecnológica en las organizaciones y la identificación de las brechas tecnológicas, a partir de los niveles de las palancas de fabricación.

\section{Metodología}

La investigación cuantitativa, de tipo descriptivo, fue desarrollada en las empresas manufactureras del sector metalmecánico de Caldas, Colombia. El área poblacional para la investigación se limitó a la región Centro Sur, que corresponde a los municipios de Manizales, Villamaría y Chinchiná, donde se concentra el $85 \%$ de la producción industrial del departamento (Centro de Pensamiento en Estrategias Competitivas, 2011). La muestra, 30 fábricas, fue definida por muestreo probabilístico, sin considerar microempresas. Las organizaciones fueron seleccionadas por conveniencia, tomando como base la participación en la mesa sectorial metalmecánica. Se incluyeron empresas de diferente tamaño, según la clasificación industrial para Colombia (Congreso de la República, 2004): $23 \%$ grandes, $43 \%$ medianas y $33 \%$ pequeñas. Los instrumentos metodológicos desarrollados por grupo de expertos del SENA y de la Universidad Autónoma de Manizales, fueron aplicados a las empresas seleccionadas mediante encuesta directa, previa realización de estudio piloto y visitas a las industrias.

\subsection{Identificación de los niveles de automatización industrial}

La identificación de los niveles de automatización existentes fue realizada partiendo de la premisa que a mayor grado de intervención humana se presenta un menor nivel de automatización y viceversa. Los niveles se identificaron en las diferentes etapas de la cadena de valor: Abastecimiento de materiales, proceso productivo, empaque y embalaje, almacenamiento y logística; también fueron evaluados en los procesos: Control y supervisión, operación, registro de información, comunicación. Los criterios de evaluación, definidos por panel de expertos, se presentan en la Tabla 1. Las empresas se clasificaron en categorías (Tabla 2), tomando como referencia los niveles de capacidad de las palancas de fabricación propuestos por Miltenburg, (2005)para empresas americanas y los estudios nacionales, Sarache et al., (2005).

\subsection{Identificación de brechas tecnológicas}

Para la identificación se consideraron tanto los 
niveles de automatización como el análisis de las diferentes palancas de fabricación: 1) Talento humano, 2) Estructura y control, 3) Gestión logística, 4) Gestión de la producción, 5) Gestión tecnológica, 6) Infraestructura; para tal efecto, se diseñó una encuesta de evaluación cerrada, basada en el diagnóstico de innovación propuesto por el Centro de Innovación y Desarrollo Empresarial de la Universidad de Cataluña (2002); algunos aspectos considerados se presentan en la Tabla 3. Además, fueron aplicadas las herramientas propuestas por Miltenburg, (2005).

Tabla 1. Criterios para evaluación de niveles de automatización

\begin{tabular}{cc}
\hline Nivel Automatización & Descripción \\
\hline 0 & $100 \%$ intervención humana, uso de los sentidos \\
25 & Uso de equipos y herramientas \\
50 & Uso de controles tipo on-off \\
75 & Uso de controles avanzados \\
100 & Uso de controles remotos \\
\hline
\end{tabular}

Tabla 2. Categorías establecidas por niveles de automatización

\begin{tabular}{|c|c|c|}
\hline Categorías & Nivel Automatización & Capacidad de fabricación \\
\hline Bajo & $<24$ & La fabricación está baja en técnica y poco cualificada. \\
\hline Medio-Bajo & $25-40$ & La fabricación consiste en rutinas y actividades, pero está baja en técnica. \\
\hline Medio & $41-55$ & $\begin{array}{l}\text { La fabricación consiste en rutinas y actividades estándar. Se satisface con mantener el } \\
\text { paso con los competidores y el estatus vigente }\end{array}$ \\
\hline Medio-Alto & $56-70$ & $\begin{array}{l}\text { El sistema de producción provee outputs cualificados, pero no se ha adoptado una } \\
\text { perspectiva a largo plazo. }\end{array}$ \\
\hline Alto & $71-85$ & $\begin{array}{l}\text { El sistema de producción provee outputs cualificados y las decisiones de fabricación } \\
\text { son consistentes con la estrategia }\end{array}$ \\
\hline Clase Mundial & $86-100$ & El sistema de producción es una ventaja competitiva. \\
\hline
\end{tabular}

Tabla 3. Aspectos considerados para la identificación de brechas tecnológicas

\begin{tabular}{|c|c|}
\hline Palancas de Fabricación & Aspectos evaluados \\
\hline 1. Talento Humano & $\begin{array}{c}\text { ¿Cómo hace progresar la gerencia el know how de la empresa en Gestión tecnológica y } \\
\text { automatización, a partir de las personas? }\end{array}$ \\
\hline 2. Estructura y Control & $\begin{array}{c}\text { ¿Qué papel juega la automatización en la planificación del negocio a largo plazo? } \\
\text { ¿Comparte la Gerencia la idea de que la automatización y la tecnología hay que gestionarla y de que } \\
\text { no se puede improvisar? }\end{array}$ \\
\hline 3. Gestión Logística & $\begin{array}{c}\text { ¿Cómo asume la gerencia el riesgo inherente a la Gestión tecnológica y automatización } \\
\text { ¿Considera la integración de la automatización a las actividades logísticas? }\end{array}$ \\
\hline 4. Gestión de la producción & $\begin{array}{c}\text { ¿Cómo es la de gestión de los procesos productivos? } \\
\text { ¿Se planifica la asignación de recursos específicos para el desarrollo de nuevos procesos de } \\
\text { producción? }\end{array}$ \\
\hline 5. Gestión de la tecnología & $\begin{array}{c}\text { ¿Qué aspectos tiene en cuenta para la automatización de procesos? } \\
\text { ¿Cómo identifica la empresa las tecnologías clave para su negocio y cómo evalúa el impacto de estas } \\
\text { tecnologías para el futuro de la organización? }\end{array}$ \\
\hline 6. Infraestructura & $\begin{array}{c}\text { ¿Existe un plan tecnológico estratégico para incorporar nuevas tecnologías al desarrollo de nuevos } \\
\text { productos, incluidas las de automatización, con una dotación presupuestaria y una estimación de la } \\
\text { rentabilidad esperada? } \\
\text { ¿Cómo es el seguimiento de las tecnologías de fabricación? } \\
\text { ¿Cuáles son las fuentes para el conocimiento de nuevas tecnologías? } \\
\text { ¿Qué criterios tienen en cuenta para la renovación o adquisición tecnológica? } \\
\text { ¿Qué actividades de vigilancia tecnológica realiza? } \\
\text { ¿Cuáles es la edad de la maquinaria? } \\
\text { ¿Cuáles son los tipos de automatización por Jerarquía de control }\end{array}$ \\
\hline
\end{tabular}




\section{Resultados y discusión}

\subsection{Niveles de automatización industrial}

Los niveles de automatización de las empresas objeto de estudio se evaluaron para las diferentes procesos y etapas de la cadena de valor; los resultados se consolidan en la Tabla 4, la cual reporta los valores medios obtenidos. Aunque en el proceso productivo estos niveles fueron clasificados como medios, en algunas etapas de la cadena de valor como: abastecimiento de materiales, almacenamiento y logística de despacho, las categorías fueron medio-bajas. Los niveles presentaron alta variabilidad como se observa en el análisis de frecuencias de la Figura 1, fluctuaron entre las categorías bajas a clase mundial. Se encontraron organizaciones con procesos completamente manuales hasta sistemas de producción con controles remotos. Con respecto al proceso productivo, se observó un mayor nivel de automatización; sin embargo, en las operaciones de empaque y embalaje, los niveles fueron medio-bajos, siguiendo la tendencia de la cadena logística.

\subsection{Análisis de las palancas de fabricación}

El análisis de frecuencias por categorías, para cada una de los aspectos considerados de las palancas de fabricación se muestra en la Figura 2. Al igual que con los niveles de automatización, se evidenció alta variabilidad en las organizaciones, con tendencia hacia valores altos. Las principales características encontradas se describen a continuación:

\subsubsection{Talento humano}

En las empresas objeto de estudio se evaluó cómo la gerencia hace progresar el know how de la empresa en gestión tecnológica y automatización, a partir de las personas. El 30\% de las organizaciones, clasificadas como de clase mundial en este aspecto, aplica un plan para el desarrollo profesional a través de la rotación interna entre diversas funciones; estimula todo tipo de actividades que promueven el aprendizaje, especialmente el trabajo en equipo y contrata y desarrolla permanentemente personal técnico calificado. E1 43\% de las organizaciones, ubicadas entre las categorías alta y media alta, contrata personal técnico calificado para incrementar la base de conocimientos de la empresa; fomenta la formación continuada de los trabajadores, pero no dispone de un plan de formación específico para cada puesto de trabajo. En las empresas ubicadas entre las categorías media y medio-baja (27\%) se promueven acciones puntuales de formación de los trabajadores y se contrata personal calificado para cubrir necesidades específicas.

\subsubsection{Estructura y control}

Para el análisis, se consideró el papel que juega la automatización en la planificación del negocio a largo plazo, la gestión de la automatización y cómo la organización asume los riesgos inherentes de la automatización.

Las empresas ubicadas en la categoría de clase mundial $(37 \%)$ realizan una planificación estratégica del negocio, teniendo en cuenta las

Tabla 4. Niveles medios de automatización industrial en las empresas del sector metalmecánico

\begin{tabular}{cccccc}
\hline Niveles de Automatización & $\begin{array}{c}\text { Abastecimiento } \\
\text { Materiales }\end{array}$ & $\begin{array}{c}\text { Proceso } \\
\text { productivo }\end{array}$ & $\begin{array}{c}\text { Empaque y } \\
\text { embalaje }\end{array}$ & Almacenamiento & $\begin{array}{c}\text { Logística } \\
\text { despacho }\end{array}$ \\
\hline Control y supervisión & 38 & 51 & 34 & 28 & 36 \\
Operación & Medio-Bajo & Medio & Medio-Bajo & Medio-Bajo & Medio-Bajo \\
& 41 & 50 & 41 & 28 & 34 \\
Registro de información & Medio & Medio & Medio & Medio-Bajo & Medio-Bajo \\
& 30 & 47 & 43 & 51 & 45 \\
Comunicación & Medio-Bajo & Medio & Medio & Medio & Medio \\
& 44 & 43 & 44 & 44 & 46 \\
& Medio & Medio & Medio & Medio & Medio \\
\hline
\end{tabular}



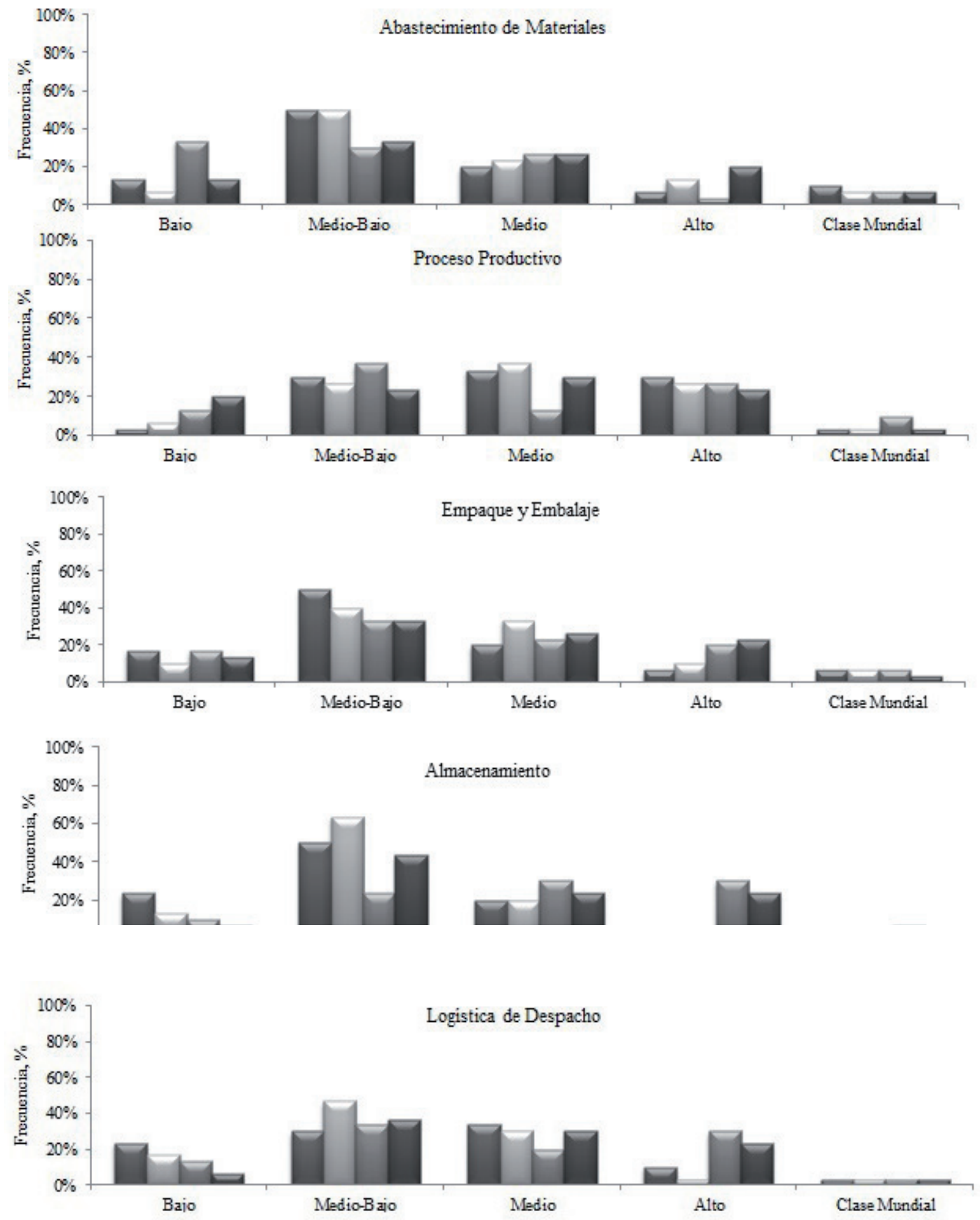

Figura 1. Niveles de automatización en las empresas del sector de metalmecánico, Caldas, Colombia. 

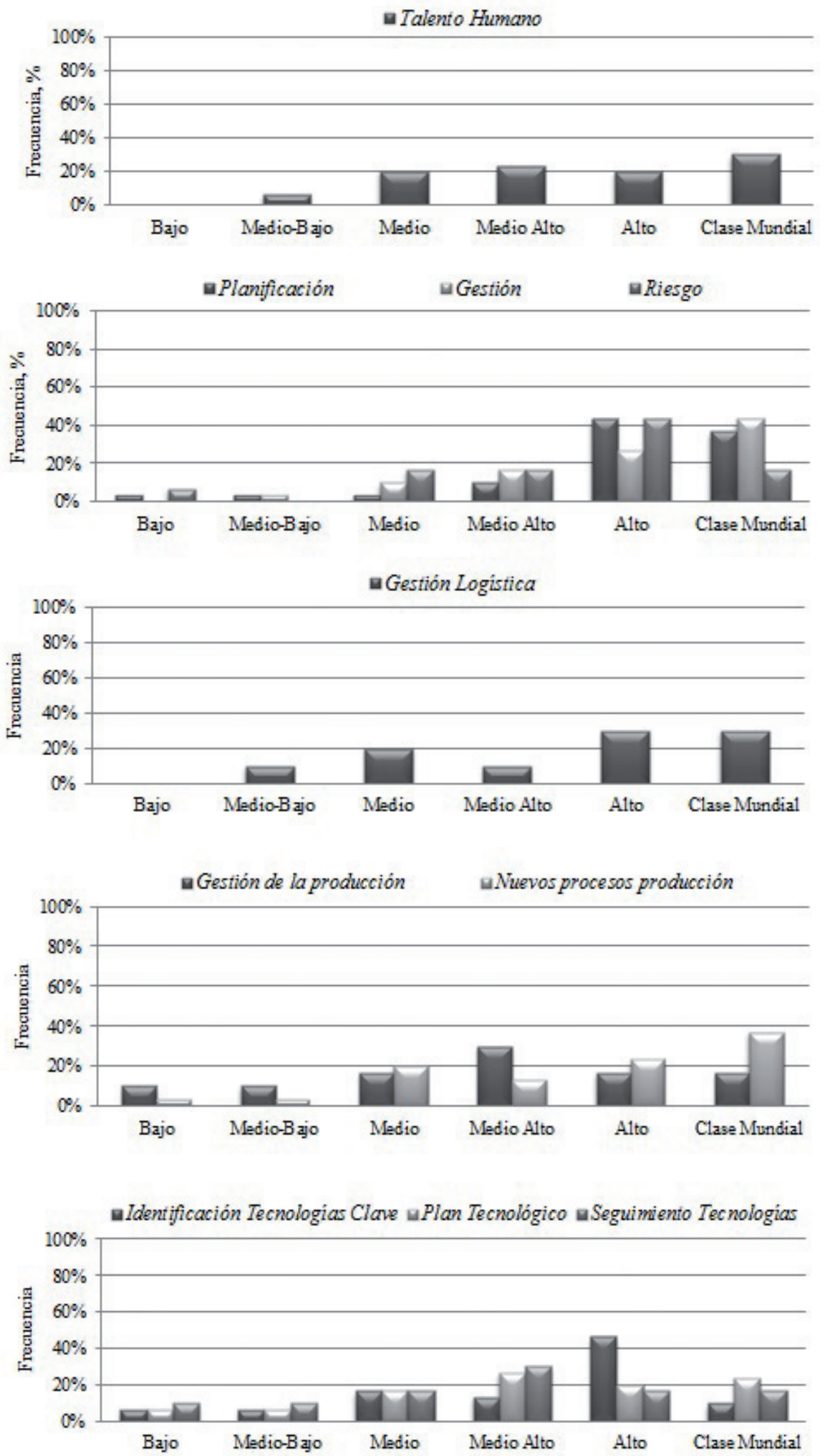

Figura 2. Análisis de frecuencia de los aspectos considerados para la identificación de brechas tecnológicas 
nuevas tendencias del mercado, lo que hacen sus competidores y las nuevas tecnologías; por tanto, tienen objetivos concretos a mediano y largo plazo y una planificación de recursos para la gestión tecnológica y la automatización. En las organizaciones clasificadas entre las categorías alta a medio-alta (53\%), la gerencia prevé cambios en el sector y realiza una planificación estratégica del negocio teniendo en cuenta estos cambios; sin embargo, no existe una vinculación directa entre esta visión estratégica y los recursos destinados a la gestión tecnológica y la automatización. Para el $10 \%$ restante, aunque la gerencia considera posibles cambios futuros, que pueden afectar a sus productos y procesos de fabricación, solo actuará cuando estos cambios ocurran.

Con respecto a la gestión de tecnología y automatización, en el $43 \%$ de las organizaciones (clase mundial), la gerencia gestiona de forma sistemática la automatización como un proceso de negocio estructurado por proyectos; se asignan recursos concretos para llevar a cabo el proceso de gestión tecnológica de forma permanente. En el $43 \%$ de las empresas clasificadas entre las categorías alta a medio-alta, el área técnica es la encargada de gestionarla. En el 14\% restante no se percibe la necesidad de disponer de una metodología para gestionar la tecnología y la automatización.

En las organizaciones de clase mundial (43\%), la gerencia se siente implicada e impulsa la gestión tecnológica y la automatización como una fuente de competitividad de la empresa, lo transmite a sus trabajadores a través de la misión, visión, política, objetivos; comunica el compromiso con la gestión tecnológica y automatización a sus clientes mediante sus argumentos de venta. En las empresas clasificadas entre las categorías alta a medio-alta (43\%), la gerencia es consciente que la gestión tecnológica y la automatización son un medio para obtener ventajas competitivas y así lo transmite a sus clientes; sin embargo, falla la comunicación a nivel interno; el compromiso no se incorpora explícitamente en los manifiestos de la dirección (misión, visión, política, objetivos). En el 10\% de las organizaciones, la gerencia de la empresa habla de gestión tecnológica e innovación, pero no consigue transmitir el mensaje de forma coherente. En el 4\% restante, la gerencia no tiene en cuenta ni menciona la gestión tecnológica y automatización en la comunicación con sus trabajadores, clientes, accionistas o proveedores.

Finalmente, en materia del riesgo, sólo el 17\% de las organizaciones, ubicadas en la categoría de clase mundial, asume riesgos tecnológicos altos y resultados a largo plazo dentro de una cartera de proyectos diversificada. En el $60 \%$ de las empresas (categoría alta a medio-alta), la gerencia promueve y potencia un clima adecuado para la gestión tecnológica y automatización a medio plazo. En el 23\% restante, se admiten riesgos limitados con resultados a mediano y corto plazo.

\subsubsection{Gestión logística}

En el 30\% de las empresas (categoría de clase mundial), las actividades logísticas se planifican integrando a los clientes y proveedores; el sistema contempla las necesidades del lanzamiento de productos innovadores, la flexibilidad, la disponibilidad y la gestión de pedidos pequeños. En el $40 \%$ de las empresas (categorías alta a medio-alta), las actividades logísticas se integran íntimamente, desde el aprovisionamiento hasta la distribución, consiguiendo que esta integración aporte valor al cliente, en términos de costos y plazos de entrega. En el 30\% restante, las actividades logísticas no se han considerado como posibles generadores de valor.

\subsubsection{Gestión de la producción}

De acuerdo con los resultados de las encuestas, los criterios para la automatización industrial, en orden de importancia, son los siguientes: 1) Aumentar la capacidad de producción, 2) Mejorar la calidad, 3) Estrategia competitiva, 4) Modernización tecnológica, 5) Flexibilidad e 6) Innovación.

En el 37\% de las empresas (categoría de clase mundial) existe un responsable con un presupuesto anual y un equipo de colaboradores internos 
y externos que tiene la misión de redefinir y mejorar los procesos productivos de acuerdo con la estrategia organizacional y con los objetivos concretos de reducción de costos, mejora de la calidad de los productos y mayor flexibilidad. En las empresas con categorías alta a medio-alta $(37 \%)$, el director de producción tiene la misión de mejorar los procesos productivos; existe un fondo para las inversiones no previstas que se puede utilizar ocasionalmente para poner en marcha algunos proyectos concretos. En el $26 \%$ restante, no existe ninguna estrategia de desarrollo de nuevos procesos de producción.

\subsubsection{Gestión de la tecnología}

Según los resultados de las encuestas y considerando el orden de importancia, los criterios para la renovación o adquisición tecnológica son: 1) Capacidad de producción, 2) Calidad, 3) Obsolescencia, 4) Flexibilidad, 5) Política Organizacional y 6) Solicitud de Clientes.

Por otra parte, las organizaciones tienen en cuenta los siguientes aspectos para la compra de tecnología: 1) Estandarización y adaptabilidad, 2) Especificaciones técnicas, 3) Precio, 4) Mantenimiento y repuestos, 5) Servicio Postventa y 6) Marca.

En el 23\% de las organizaciones (categorizadas como clase mundial), existe un plan tecnológico a mediano y largo plazo, coherente con las necesidades futuras de mercado y la estrategia de empresa; se asigna un responsable con un presupuesto para su ejecución; periódicamente, se evalúa el desarrollo del plan y se introducen las modificaciones necesarias. En el $47 \%$ de las empresa (categorías alta a media-alta aunque existe un plan tecnológico, no se aplica, debido a las presiones del día a día; sin embargo, se intenta que los nuevos productos incorporen las tecnologías más avanzadas y se hace una previsión de los recursos financieros necesarios para abordar el proyecto. En un $23 \%$ de las organizaciones (categoría media y media-baja) no existe ningún plan tecnológico concreto; se intentan identificar las tecnologías y obtener los recursos necesarios por proyecto. En el 7\% restante (categoría baja), no existe ningún plan estratégico para incorporar nuevas tecnologías, ni previsiones de adjudicación de recursos financieros con esta finalidad.

Sólo en el 10\% de las organizaciones (clase mundial), la vigilancia y la prospectiva tecnológica son actividades integradas en la gestión de la empresa, en la cual se han detectado los conocimientos y competencias clave y se replantea periódicamente como mejorarlos. En el $60 \%$ de las organizaciones (ubicadas entre las categoría alta a medio-alta) se realizan algunas actividades como seguimiento de la competencia, participación en ferias y congresos nacionales e internacionales, contacto con algunas fuentes de conocimiento próximas (universidades y expertos); reuniones internas para analizar la forma de incorporarlos a la empresa y se han identificado algunos conocimientos y competencias clave. Un $23 \%$ de las empresas realiza el seguimiento con la asistencia a ferias nacionales. El 7\% restante cree que no necesita tener de forma explícita ningún mecanismo de vigilancia tecnológica y no tiene identificados sus conocimientos ni competencias claves.

Para la redefinición de los procesos productivos y la innovación, el 17\% de las empresas (clase mundial) tiene mecanismos de benchmarking que les permite conocer las tecnologías de producción de las empresas líderes en el panorama mundial. En el $47 \%$ de las organizaciones (categorías alta a medio-alta), la lectura de revistas especializadas, la asistencia a cursos, congresos y ferias son los mecanismos de acceso para explorar alternativas tecnológicas de los procesos productivos implantados. El 10\% de las empresas no cuenta con mecanismos de seguimiento de las tecnologías de fabricación utilizadas por las empresas del sector.

\subsubsection{Infraestructura}

Laparticipaciónporcentualporjerarquíade control, de los tipos o mecanismos de automatización existentes fue identificada de la siguiente manera en las empresas objeto de estudio: 1) 
Actuadores: mecánicos 39\%, neumáticos 29.1\%, hidráulicos $23.4 \%$ y servomecánicos $8.5 \%$. 2) Sensores: eléctricos $60 \%$, proporcionales $20.4 \%$, inteligentes $9.3 \%$, otros $10.3 \%$. 3) Controladores: PLC $45.5 \%$, PAC $24.7 \%$, Arquitectura integrada $14 \%$, microprocesadores $8 \%$, otros $7.8 \%$. 4) Sistema de comunicación: redes $35.7 \%$, bus de campo $32.6 \%$, software de supervisión $20.7 \%$, ERP $7.5 \%$, otros $3.5 \%$.

El $10 \%$ de las organizaciones tiene alguna aplicación de robótica; las demás $(90 \%)$ no la tienen implementada. Por otra parte, el análisis de madurez y obsolescencia tecnológica se realizó teniendo en cuenta la edad de los equipos de automatización con los siguientes resultados: 0-5 años: $20 \%$; 6-10 años: 42\%; 11-20 años: $25 \%$; > 20 años: $13 \%$.

\subsection{Brechas tecnológicas en automatización industrial}

Las brechas en automatización industrial para cada uno de los aspectos considerados se presentan en la Figura 3. De acuerdo con el grado de intervención humana, se tienen niveles medios de automatización (40\%); sólo el 10\% se encuentra entre las categorías media-alta a clase mundial; en el $50 \%$ restante, se presenta una mayor brecha tecnológica, por lo bajos niveles de automatización encontrados, dado el carácter manual de las operaciones de la cadena de valor.
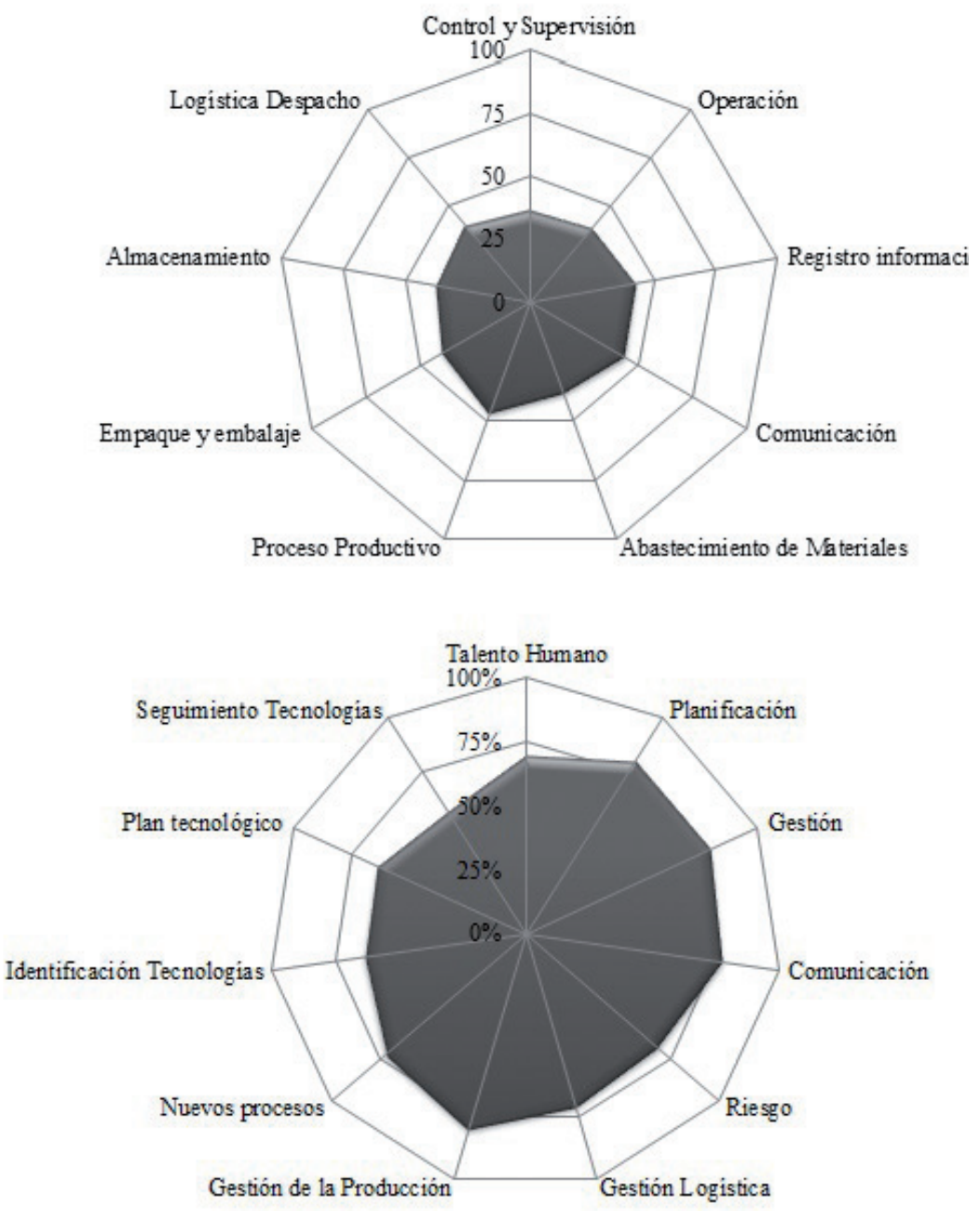

Figura 3. Identificación de brechas en automatización sector metalmecánico, Caldas, Colombia 
Los procesos se encuentran definidos por una distribución acompasada por operario, debido al carácter manual de algunas etapas, en especial el empaque, el embalaje y la gestión logística, que comprende las operaciones de abastecimiento de materiales, almacenamiento y despacho. Con respecto a los aspectos organizacionales, en la Figura 3 se visualiza una reducción en la brecha; se evidencian oportunidades de mejora en la gestión tecnológica, en la identificación y seguimiento de nuevas tecnologías de automatización y en los planes tecnológicos y de automatización.

\section{Conclusiones}

Las mayores brechas tecnológicas en automatización industrial en los procesos de la cadena de valor de las empresas del sector metalmecánico de Caldas se tienen en la gestión logística que comprende las operaciones de abastecimiento de materiales, almacenamiento y despacho, las cuales presentaron menores niveles de automatización.

Fortalecer la gestión tecnológica en las organizaciones, en especial, la formulación y desarrollo de planes tecnológicos y el seguimiento de nuevas tecnologías puede contribuir al cierre de las brechas detectadas.

La articulación con Centros de Investigación y Desarrollo Tecnológico y Universidades puede facilitar el desarrollo de tecnologías accesibles a la organización, que cumplan con los estándares de adaptabilidad y estandarización, los cuales son claves dado que la automatización debe integrarse a la tecnología existente. Mejorar los niveles de automatización en el 50\% de las empresas objeto de estudio puede reducir la brecha tecnológica detectada.

\section{Referencias Bibliográficas}

Cámara de Comercio de Bogotá. (2005). Caracterización de las cadenas productivas de manufactura y servicios de Bogotá y Cundinamarca. Bogotá, Colombia.

Centro de pensamiento en estrategias competitivas.
(2011). Diagnóstico de temáticas CAF para Manizales. Universidad del Rosario, Facultad de Ciencias Políticas y Relaciones Internacionales, Bogotá, Colombia.

CIDEM (Centro de Innovación y Desarrollo Empresarial). (2002). Guía de gestión de la innovación. Universidad de Cataluña, Barcelona, España.

Congreso de la República. (2004). Ley 905-2004. Promoción del desarrollo de la micro, pequeña y mediana empresa colombiana. Bogotá, Colombia.

DNP (Departamento Nacional de Planeación). (2007). Agenda Interna para la Productividad y la Competitividad. Documento sectorial, Metalmecánica y Siderurgia. Bogotá, Colombia.

DNP (Departamento Nacional de Planeación). (2008). CONPES 3582- Politica Nacional de Ciencia, Tecnología e Innovación. Bogotá, Colombia.

DNP (Departamento Nacional de Planeación). (2009). CONPES 3527- Política Nacional de Competitividad y Productividad. Bogotá, Colombia.

DNP (Departamento Nacional de Planeación) \& Colciencias. (2005). Plan Estratégico del Programa Nacional de Desarrollo Tecnológico, Industrial y Calidad, 2005-2015. Bogotá, Colombia.

DNP (Departamento Nacional de Planeación) \& Colciencias. (2000). Plan Estratégico del Programa Nacional de Desarrollo Tecnológico Industrial y Calidad 2000 - 2010. Bogotá, Colombia.

González, García \& Montoya. (2004). Evaluación y Determinación del Perfil Tecnológico de las empresas del sector Metalmecánico de Pereira y Dosquebradas. Sciencia et Technica Año X (25), 221-226.

Huerta, Navas \& Almodóvar. (2004). La Diversificación desde la Teoría de Recursos 
y Capacidades. Cuadernos de estudios empresariales (14), 87-104.

López, N. A. (2005). Identificación de estrategias de mercados meta de los sectores metalmecánico, textil, confecciones y alimentos de la ciudad de Manizales. Tesis, Universidad Nacional de Colombia sede Manizales, Manizales, Colombia.

Medina, Franco, Landinez \& Aguilera. (2010). Modelo de Prospectiva y Vigilancia Tecnológica SENA. Centro de Vigilancia y Prospectiva Tecnológica Universidad del Valle, Cali, Colombia.

Miltenburg, J. (2005). Manufacturing strategy: How to formulate and implement a winning plan. New York: Taylor \& Francis, Inc.

Moreno, G. (2001). Automatización de procesos Industriales. México: Editorial Alfa Omega.

Observatorio de Mercado de Trabajo. (2010). Estudio sector metalmecánico área metropolitana de Bucaramanga. Bucaramanga, Colombia.

Ortiz \& Nagles. (2008) Gestión de Tecnología e
Innovación. Bogotá: Universidad EAN.

Piedrahita, R. (2001). Ingeniería de la automatización industrial. Bogotá: Editorial Alfa Omega.

Pineda \& Jara. (2009). Prospectiva y Vigilancia Tecnológica en la Cadena Fibra-TextilConfecciones. Universidad del Rosario, Bogotá, Colombia.

Ruedas, C. (2010). Automatización Industrial: Áreas de aplicación en la Ingeniería. Guatemala: Universidad Rafael Landivar.

Sarache, Cárdenas \& Giraldo. (2005). Procedimiento para la definición y jerarquización de prioridades competitivas de fabricación: aplicaciones en la industria metalmecánica. Revista Ingeniería y Competitividad., 7 (2), 84-91.

SENA (Servicio Nacional de Aprendizaje). (2002). Caracterización Ocupacional del Sector Metalmecánico. Manizales. Manizales: Servicio Nacional de Aprendizaje, Manizales, Colombia. 